\title{
A Case Report of Thyroid Carcinoma Confined to Ovary and Concurrently Occult in the Thyroid: Is Conservative Treatment Always Advised?
}

\author{
Nunzia Brusca ${ }^{1}$; Susanna Carlotta Del Duca ${ }^{1}$; Rita Salvatori ${ }^{2}$; Antonio D’Agostini ${ }^{2}$; Pina \\ Cannas $^{2}$; Maria Giulia Santaguida ${ }^{1}$; Camilla Virili ${ }^{1}$; Loredana Bianchi ${ }^{3}$; Lucilla Gargano ${ }^{3}$; \\ Marco Centanni ${ }^{1,3,}$ \\ ${ }_{2}^{1}$ Department of Medico-Surgical Sciences and Biotechnologies, "Sapienza" University of Rome, Latina, Italy \\ ${ }_{3}^{2}$ Department of Nuclear Medicine, Santa Maria Goretti Hospital, AUSL Latina, Latina, Italy \\ ${ }^{3}$ Endocrinology Unit, Santa Maria Goretti Hospital, AUSL Latina, Latina, Italy \\ ${ }^{*}$ Corresponding author: Marco Centanni, Department of Medico-Surgical Sciences and Biotechnologies, “Sapienza” University of Rome, Latina, Italy. Tel/Fax:+39-0649972604, E-mail: \\ marco.centanni@uniroma1.it
}

Received: February 17, 2014; Revised: April 22, 2014; Accepted: May 10, 2014

\begin{abstract}
Introduction: Struma ovarii is an ovarian teratoma, represented in more than $50 \%$ by thyroid tissue. Five percent of struma ovarii cases have been proven to be malignant and, as in the thyroid gland, papillary thyroid carcinoma is the most common histotype arising in struma ovarii. Because of the unusual occurrence of this tumor, its management and follow-up after pelvic surgery is still controversial. Usually, total thyroidectomy followed by radioiodine treatment is the choice treatment in metastatic malignant struma ovarii, while these procedures are still controversial in non-metastatic thyroid cancer arising in struma ovarii.

Case Presentation: We report a female with follicular variant of papillary thyroid carcinoma arising in struma ovarii. After pelvic surgery, thyroid morphofunctional examinations were performed and a single nodular lesion in the left lobe was discovered. The patient underwent total thyroidectomy and histological examination showed a papillary carcinoma. Radioiodine-ablation of residual thyroid tissue was performed and levothyroxine mildly-suppressive treatment was started.

Conclusions: Amore aggressive treatment should not be denied for malignant struma ovarii without any evidence, even when apparently confined into the ovary. However, in selected cases, aggressive treatment may be advisable to decrease the risk of recurrence and to allow an accurate follow-up.
\end{abstract}

Keywords:Thyroid Carcinoma; Thyroidectomy; Struma Ovarii

\section{Introduction}

Germ cell tumors represent $15-20 \%$ of ovarian cancers and most of them are mature cystic teratomas (1). Struma ovarii is a highly specialized ovarian teratoma, chiefly represented by thyroid tissue (2). In fact, while 5-15\% of teratomas contains small foci of thyroid tissue, this latter should represent more than $50 \%$ in a struma ovarii (1). Approximately, $5 \%$ of struma ovarii has been proven to be malignant and metastases are uncommon (2). Fairly good prognosis characterizes these patients: survival rate of patients in follow-up at 5, 10 and 25 years is $92 \%$, $85 \%$ and $79 \%$, respectively (3). Long-term follow-up is recommended in all cases (4). As in the thyroid, papillary carcinoma is the most common malignant histotype (70\%, 1/3 represented by its follicular variant) arising in struma ovarii; while follicular carcinoma is observed in the remaining 30\% (3-5). Malignant struma ovarii generally occurs in patients in the fifth and sixth decades of life (5), it is unilateral (94\%) and more frequently involves the left ovary $(3,5)$. Functional thyroid alterations are unusual and overt hyperthyroidism is reported in $5-8 \%$ of cases
(5). The most common clinical presentation of struma ovarii is a pelvic mass (3); lower abdominal pain, abnormal vaginal bleeding or menstrual irregularities and ascites may be associated (5). However, the tumor is often unexpectedly diagnosed during abdominal/pelvic ultrasound or surgery $(3,5)$. Abdominal hysterectomy and bilateral salpingo-oophorectomy with omentectomy is the surgical treatment for malignant struma ovarii. Unilateral salpingo-oophorectomy is the preferred treatment for women who require preservation of fertility. Being this tumor fairly uncommon, there is a lack of diagnostic and treatment guidelines (4, 6-8). After initial surgery, some authors suggest that the management of malignant struma ovarii may be similar to that of other germ cell tumors (1); other authors suggest, as in differentiated cancer of thyroid gland management, to perform a thyroidectomy followed by radiotherapy with 131 isotope of Iodine (RAI) (9) and levothyroxine suppressive therapy (3, $4,7,8)$, although restricted to patients with recurrence or residual disease $(2,4,5)$. No consensus has been reached

Copyright (C) 2015, Research Institute For Endocrine Sciences and Iran Endocrine Society. This is an open-access article distributed under the terms of the Creative Commons Attribution-NonCommercial 4.0 International License (http://creativecommons.org/licenses/by-nc/4.0/) which permits copy and redistribute the material just in noncommercial usages, provided the original work is properly cited. 
in performing prophylactic total thyroidectomy and the treatment strategy for nonmetastatic malignant disease remains controversial. We present a case of 30-year-old female with follicular variant of papillary thyroid carcinoma arising in struma ovarii and concomitant differentiated cancer of thyroid gland.

\section{Case Presentation}

A 30-year-old woman was admitted to the emergency room because of persistent abdominal pain in the right side, appeared some day before, during a shift work as agricultural laborer. An accurate medical history ascertained that she had an increased steady weight $(109 \mathrm{~kg}$; $\left.\mathrm{BMI}=40 \mathrm{Kg} / \mathrm{m}^{2}\right)$, but she was in apparently good health also showing customary appetite and regular menses, before the appearance of pain. Physical examination revealed the presence of a large mass, palpable in hypogastric region and in right lower abdominal quadrant. Ultrasonography confirmed an abdominal mass adjacent to the right ovary. To better define the diagnosis, the physician required blood sampling for routine tests, gynecological counseling and an abdominal computer tomography (CT). The abdominal CT confirmed the presence of a mass measuring $10 \times 6 \mathrm{~cm}$, in the right ovary. The patient underwent right unilateral salpingo-oophorectomy. In particular, an ovariian cyst $(7,3 \times 3 \mathrm{~cm})$ along with a portion of the fallopian tubes of $4 \mathrm{~cm}$ and the vermiform appendix were removed. Peritoneal washing and multiple peritoneal biopsies in nearby areas (Douglas, bladder, bilateral paracolic gutter, and diaphragm) were also performed. Histological examination revealed the presence of a follicular variant of papillary thyroid carcinoma (0.9 $\mathrm{cm}$ ) harbored in a mature teratoma. The cancer was localized and the right ovary capsule was not infiltrated by the tumor. Based on Union for International Cancer Control (UICC) 2002, classification tumor received a pT1a grading. Appendix showed the features of chronic follicular inflammation and the remaining tissues were disease-free. After surgery, functional examination of thyroid gland revealed serum iodothyronines levels in the normal range, thyroid ultrasonography showed a normal glandular volume with patchy echo-structure and, in the left thyroid lobe, the presence of a 23, $4 \times 14 \times 9 \mathrm{~mm}$ hypoechogenic nodular lesion. An ultrasound assisted fine needle aspiration of this thyroid lesion was performed and cytological examination showed the presence of dense colloid in particles and small groups of thyrocytes with notes of oxyphilic metaplasia and anisonucleosis; this sample was classified as follicular lesion with oxyphilic cells of undetermined significance (category III according to the Bethesda system, 2009) (10). So far, in presence of follicular lesion and for an appropriate follow-up of papillary carcinoma arising in struma ovarii, the patient underwent total thyroidectomy $(11,12)$. Histological examination revealed that the nodule examined in the left lobe was benign, but showed a papillary micro-carcinoma in the right lobe (diameter $=0.2 \mathrm{~cm}$; pT1a) within a hyperplastic thyroid characterized by abundant oxyphil cells. The ablation of residual thyroid tissue was performed by a dose of $30 \mathrm{mCi}$ of $131 \mathrm{I}$ according to Meas et al.(13). Whole body scan after therapeutic dose showed two focal areas of strong uptake in the anterior region of the neck, in right superior paramedian and left inferior paramedian areas, due to minimal glandular remnants; no area of uptake in the pelvis or elsewhere was notice. The thyroidstimulating hormone (TSH) value before radioiodine therapy was $64.8 \mathrm{mU} / \mathrm{L}$ and serum thyroglobulin ( $\mathrm{Tg}$ ) was $1.24 \mathrm{mg} / \mathrm{dL}$, in the absence of anti-thyroglobulin antibodies (anti-TgAb). Levothyroxine treatment was started (1.6 $\mu \mathrm{g} / \mathrm{Kg} / \mathrm{day})$ and thyroid hormones and TSH were evaluated after four months. Levothyroxine dose had been increased until an appropriate TSH value $(0.24 \mathrm{mU} / \mathrm{L})$ had reached at $1.92 \mu \mathrm{g} / \mathrm{Kg} /$ day. Six months after total thyroidectomy, patient underwent neck ultrasonography and $\mathrm{Tg}$ evaluation, which showed no evidence of recurrences. Patient is up to now in follow-up for both struma ovarii and differentiated thyroid cancer according to European Thyroid Association guidelines (12).

\section{Discussion}

First described at the end of 21th century, struma ovarii is a rare tumor representing less than $1 \%$ of all ovarian tumors $(6,14)$ and $2.7 \%$ of all dermoid tumors $(6)$. A diagnostic and therapeutic characterization of struma ovarii is needed as it may harbor a differentiated thyroid cancer (1). Even the generic term "malignant struma ovarii" has been, in fact, replaced with the more appropriate "thyroid carcinoma arising in struma ovarii" (TCASO), namely when histological features support the existence of a well-differentiated thyroid carcinoma $(3,14,15)$. Molecular evidence that malignant struma ovarii and differentiated thyroid cancer may share similar pathogenic events $(3,16)$ has been presented. Approximately $70 \%$ of follicular cell-derived thyroid carcinomas are associated with activating mutations of BRAF, RAS, RET and NTRK1 $(3,4,16)$. BRAF mutations have been described in 29-69\% of primitive papillary thyroid cancer and in particular V600E missense mutation type is the most common alteration in sporadic papillary carcinoma $(3,17)$. Also in four out of six malignant struma ovarii and in none of nine benign struma ovarii, BRAF mutations were observed in the Schmidt's study $(3,4)$. The concurrent presence of the same genetic alteration (i.e. BRAF mutations), in thyroid neoplasia and in struma ovarii would be intriguing in that it may support the hypothesis of a multifocal thyroid neoplasia in two distant sites $(13,16)$. Autonomous histopathological criteria have not been established for thyroid carcinoma arising in struma ovarii and, so far, the diagnosis of malignant struma ovarii follows the guidelines for the diagnosis of primary thyroid carcinoma (3). Evidence also indicates that patients with malignant struma ovarii should be treated as those hav- 
ing differentiated cancer in the thyroid gland $(3,10)$.

In patients with thyroid cancer confined to the ovary, pelvic surgery has been considered sufficient and prophylactic thyroidectomy not recommended $(1,2,14)$; in these patients follow-up ensues as in non-aggressive DTC $(1,14)$. Fine needle aspiration cytology has been considered appropriate when thyroid nodular lesions would have been detected at ultrasound $(1,4,14)$. Noticeably, however, a thyroid carcinoma concurrent with malignant struma ovarii is increasingly detected $(14,18)$ and the treatment of this association is, as yet, not properly defined. In fact, a more extensive treatment, e.g. total thyroidectomy and RAI, after pelvic surgery, is only recommended for malignant struma ovarii with extra-ovarian spread or distant metastasis (1-3). In patients with extra-ovarian or metastatic disease, evidence suggests that radioiodine ablation and thyrotropin suppression are associated with increased disease-free survival $(3,7)$. According to another study, patients who underwent total thyroidectomy and radio ablation after pelvic surgery were free of recurrence even after 36 years $(7,8)$. On the contrary, a recurrence rate of $50 \%$ was associated with the conservative treatment of patients (8). Moreover, a delayed appearance of distant metastasis has been described three years after the treatment in patients with struma ovarii who underwent pelvic surgery alone (18). A not negligible fraction (6\%) of patients with longer follow up, developed distant metastases after one, three and seven years (15). Two-thirds of metastasis occurred at the time of disease recurrence, rather than at first presentation (7); in fact, a number of cases were diagnosed as malignant only following the detection of metastatic recurrences (7). In this clinical case, an occult papillary microcarcinoma concomitant to malignant struma ovarii has been described. High prevalence of occult thyroid papillary microcarcinoma has been described in autoptic studies (36\%) and/ or following surgery for benign conditions (2-24\%) (19). However, some of these microcarcinomas may represent more aggressive varieties of disseminated tumor cells (DTCs) (17). Indeed, papillary microcarcinoma, although infrequently, may show significant differences in clinical behavior, sometimes being very aggressive, despite small primary tumor size (17). According to Meas et al. (13), the contemporary presence of two possibly related thyroid cell-derived tumors, although with low risk stratification, may be considered as a multifocal expression of the same pathogenic alteration. They suggested that a threshold of more than one centimeter as sum of all foci may require a more cautious seeking of thyroid tissue lesions (13). In this view, American Thyroid Association guidelines (20) concerning DTC follow-up should be reinterpreted as being the struma ovarii and papillary carcinoma (any size)a multifocal expression of the same tumor. In conclusion, a treatment including thyroidectomy followed by radioablation should not be denied a priori, but in selected cases may be advisable to decrease the risk of recurrence and to allow an accurate follow-up (7).

\section{Authors' Contributions}

Study concept and design: Brusca, Del Duca; acquisition of data: Salvatori, Cannas, D’Agostini, Bianchi, Santaguida; analysis of the manuscript: Gargano, Virili; critical revision of the manuscript for important intellectual content: Gargano, Santaguida, Del Duca; statistical analysis: $\mathrm{N} / \mathrm{A}$; Administrative, technical and material support: N/A; study supervision: Centanni.

\section{Funding/Support}

This study was supported by Dept of Medico-Surgical Sciences and Biotechnologies, "Sapienza" University of Rome and Endocrinology Unit, Santa Maria Goretti Hospital.

\section{References}

1. Kabukcuoglu F, Baksu A, Yilmaz B, Aktumen A, Evren I. Malignant struma ovarii. Pathol Oncol Res. 2002;8(2):145-7.

2. McGill JF, Sturgeon C, Angelos P. Metastatic struma ovarii treated with total thyroidectomy and radioiodine ablation. Endocr Pract. 2009;15(2):167-73.

3. Zhang X,Axiotis C. Thyroid-type carcinoma of struma ovarii.Arch Pathol Lab Med. 2010;134(5):786-91.

4. Salman WD, Singh M, Twaij Z. A case of papillary thyroid carcinoma in struma ovarii and review of the literature. Patholog Res Int. 2010;2010:352476.

5. Hatami M, Breining D, Owers RL, Del Priore G, Goldberg GL. Malignant struma ovarii--a case report and review of the literature. Gynecol Obstet Invest. 2008;65(2):104-7.

6. Yoo SC, Chang KH, Lyu MO, Chang SJ, Ryu HS, Kim HS. Clinical characteristics of struma ovarii.J Gynecol Oncol. 2008;19(2):135-8.

7. Jean S, Tanyi JL, Montone K, McGrath C, Lage-Alvarez MM, Chu CS. Papillary thyroid cancer arising in struma ovarii.J Obstet Gynaecol. 2012;32(3):222-6.

8. Barrera JR, Manalo LA, Ang FL. Papillary thyroid-type carcinoma arising from struma ovarii. BMJ Case Rep. 2012;2012.

9. Alvarez DM, Lee V, Bhatt S, Dogra VS. Struma ovarii with papillary thyroid carcinoma. J Clin Imaging Sci. 2011;1:44.

10. Cibas ES, Ali SZ, N. C. I. Thyroid FNA State of the Science Conference . The Bethesda System For Reporting Thyroid Cytopathology. Am J Clin Pathol. 2009;132(5):658-65.

11. Danese D, Centanni M, Farsetti A, Andreoli M. Diagnosis of thyroid carcinoma.J Exp Clin Cancer Res. 1997;16(3):337-47.

12. Pacini F, Schlumberger M, Dralle H, Elisei R, Smit JW, Wiersinga $\mathrm{W}$, et al. European consensus for the management of patients with differentiated thyroid carcinoma of the follicular epithelium. EurJ Endocrinol. 2006;154(6):787-803.

13. Meas T, Vercellino L, Faugeron I, Toubert ME. The 2009 revised American Thyroid Association guidelines for thyroid cancer: multifocality in T1 tumors in question, for or against a more minimalist approach? Thyroid. 2013;23(8):1042-3.

14. Marti JL, Clark VE, Harper H, Chhieng DC, Sosa JA, Roman SA. Optimal surgical management of well-differentiated thyroid cancer arising in struma ovarii: a series of 4 patients and a review of 53 reported cases. Thyroid. 2012;22(4):400-6.

15. Collins AM, Bodenner D, Chen C, Stone P, Stack BC, Jr.. Delayed treatment of papillary thyroid carcinoma arising from struma ovarii in a patient with history of bilateral salpingo-oophorectomy: a case report. Endocr Pract. 2012;18(1):e1-4.

16. Coyne C, Nikiforov YE. RAS mutation-positive follicular variant of papillary thyroid carcinoma arising in a struma ovarii. Endocr Pathol. 2010;21(2):144-7.

17. Ardito G, Revelli L, Giustozzi E, Salvatori M, Fadda G, Ardito F, et al. Aggressive papillary thyroid microcarcinoma: prognostic factors and therapeutic strategy. Clin Nucl Med. 2013;38(1):25-8.

18. Janszen EW, van Doorn HC, Ewing PC, de Krijger RR, de Wilt JH, Kam BL, et al. Malignant struma ovarii: good response after thy- 
roidectomy and I ablation therapy. Clin Med Oncol. 2008;2:147-52.

19. Sakorafas GH, Giotakis J, Stafyla V. Papillary thyroid microcarcinoma: a surgical perspective. Cancer Treat Rev. 2005;31(6):423-38.

20. American Thyroid Association Guidelines Taskforce on Thyroid
N, Differentiated Thyroid C, Cooper DS, Doherty GM, Haugen BR, Kloos RT, et al. Revised American Thyroid Association management guidelines for patients with thyroid nodules and differentiated thyroid cancer. Thyroid. 2009;19(11):1167-214. 